\title{
Puntos de corte provisionales para el diagnóstico de sarcopenia en ancianos de Caldas, Colombia
}

\author{
Johan Sebastián Villada-Gómez ${ }^{1,2}$, Clara Helena González-Correa ${ }^{1,3}$, Felipe Marulanda-Mejía ${ }^{2}$ \\ 1 Grupo de Investigación en Bioimpedancia Eléctrica, Universidad de Caldas, Manizales, Colombia \\ 2 Grupo de Investigación en Gerontología y Geriatría, Universidad de Caldas, Manizales, Colombia \\ 3 Grupo de Investigación en Nutrición, Metabolismo y Seguridad Alimentaria, Universidad de Caldas, Manizales, \\ Colombia
}

Introducción. La sarcopenia es la disminución de masa muscular y la consecuente reducción del rendimiento y de la función física. Su aparición puede ser indicativa de riesgo de otras condiciones en los ancianos, y su diagnóstico requiere la comparación con los datos sobre la composición corporal de la población joven sana para establecer valores de referencia de la disminución de la masa muscular. Objetivo. Establecer puntos provisionales de corte recolectados mediante bioimpedancia eléctrica para el diagnóstico de sarcopenia en ancianos caldenses a partir de los datos de la composición muscular esquelética de jóvenes.

Materiales y métodos. Se utilizaron las mediciones de la composición corporal de jóvenes sanos entre los 18 y los 35 años de edad tomadas mediante bioimpedancia eléctrica con base en las cuales se estableció el índice de masa muscular esquelética. Se aplicó la prueba de Kolmogorov-Smirnov para evaluar la normalidad de los datos y se estableció el promedio de dicho índice con sus desviaciones estándar tanto para hombres como para mujeres.

Resultados. A partir de estos datos, se estableció como bajo un índice de masa muscular esquelética con un valor de dos desviaciones estándar por debajo de la media del índice en jóvenes. En las mujeres el valor fue de $6,42 \mathrm{~kg} / \mathrm{m}^{2} \mathrm{y}$, en hombres, de $8,39 \mathrm{~kg} / \mathrm{m}^{2}$.

Conclusión. Estos valores son similares a los determinados por algunos autores en población asiática, lo que evidencia que esta tiene características de composición corporal similares a la población de estudio. Hasta donde se sabe, este es el primer estudio que ofrece una descripción de la composición corporal muscular esquelética en jóvenes colombianos y establece puntos de corte para el diagnóstico de sarcopenia en ancianos de esta región centro-andina del país.

Palabras clave: sarcopenia; músculo esquelético; envejecimiento; impedancia eléctrica; composición corporal; Colombia.

doi: https://doi.org/10.7705/biomedica.v38i4.4302

\section{Provisional cut-off points for the diagnosis of sarcopenia in elderly people from Caldas (Colombia)}

Introduction: Sarcopenia is defined as a decrease in muscle mass associated with the reduction of performance and physical function. It is an indicator of adverse outcomes in elderly people. Its diagnosis requires body composition data of healthy young population to establish reference values of low muscle mass.

Objective: To establish a provisional cut-off point for sarcopenia in the elderly population from Caldas based on the skeletal muscle composition data of young people using electric bioimpedance.

Materials and methods: Body composition data from healthy young people (18 to 35 years old) were used to calculate the skeletal muscle mass index. The Kolmogorov-Smirnov test was applied to evaluate the normality of the data and then the average and standard deviation of muscle mass index were established for both men and women.

Results: Based on these data, we established as low muscle mass a skeletal muscle mass index of two standard deviations below the mean of the muscular mass index in young people. The cut-off points were $6.42 \mathrm{~kg} / \mathrm{m}^{2}$ and $8.39 \mathrm{~kg} / \mathrm{m}^{2}$ for women and men, respectively.

\section{Contribución de los autores:}

Todos los autores contribuyeron a la concepción y el diseño del estudio; la adquisición, análisis e interpretación de los datos, y a la escritura del manuscrito. 
Conclusion: This is the first study that offers a description of skeletal muscle body composition in young Colombians and provides cut-off points for the diagnosis of sarcopenia in the elderly population from the Central-Andean region. The data gathered are similar to those proposed by some Asian authors, which shows that the population of this Colombian region has body composition characteristics similar to those from Asia.

Key words: Sarcopenia; muscle, skeletal; aging; electrical impedance; body composition; Colombia. doi: https://doi.org/10.7705/biomedica.v38i4.4302

Al envejecer ocurren cambios en la composición corporal, especialmente en la masa muscular (1). En diversos estudios longitudinales, se ha establecido que a partir de los 35 años de edad hay una disminución progresiva de la masa, la fuerza y el poder muscular esquelético (2-8), proceso que se denomina sarcopenia.

En el consenso del European Working Group on Sarcopenia in Older People (EWGSOP) de 2010 (9), se definió la sarcopenia como un síndrome multifactorial prevalente en ancianos y caracterizado por la pérdida de masa muscular involuntaria y no asociada a alguna enfermedad, con disminución de la fuerza, del rendimiento muscular y, al cabo, de la capacidad funcional (2), todo ello asociado con una peor calidad de vida y consecuencias adversas en ancianos $(5,10,11)$.

La medición de la masa muscular puede hacerse con diferentes métodos, algunos directos, como la medición de la creatinina en orina a lo largo de 24 horas, y otros indirectos, como la absorciometría con rayos $X$ de energía dual, la tomografía computadorizada, las imágenes de resonancia magnética, el análisis de bioimpedancia eléctrica o la medición del potasio corporal total $(5,12)$. En las investigaciones, las imágenes de resonancia magnética y la tomografía computadorizada se consideran las técnicas de referencia, seguidas por la absorciometría con rayos $\mathrm{X}$ de energía dual (13). Sin embargo, son costosas y de disponibilidad limitada en la práctica clínica, en tanto que el análisis de bioimpedancia eléctrica es una técnica portátil y segura, más asequible y económica, y de suficiente precisión para estimar la masa muscular esquelética en los adultos, además de ser apropiada para su uso en grandes cohortes (12-14).

Correspondencia:

Johan Sebastián Villada-Gómez, Calle 67 №38A-56, Manizales, Colombia

Teléfono: (576) 8912801

josevigo92@gmail.com
A pesar de que se han intentado estandarizar una definición y unos criterios operativos para el diagnóstico de disminución de la masa muscular, aún existen muchos vacíos. En el consenso EWGSOP y en los de otros autores, se recomienda establecer los valores de referencia en cada región con base en una población normal (adultos jóvenes sanos), y que el punto de corte se sitúe dos desviaciones estándar por debajo del valor medio de la población de referencia $(9,15,16)$. En este sentido, para el diagnóstico de sarcopenia en ancianos se requieren los datos de la composición muscular en jóvenes sanos. En la región de estudio, se desconoce la composición corporal muscular de la población adulta joven, lo que impide establecer los puntos de corte para la disminución de la masa muscular en los ancianos.

El objetivo de este estudio fue establecer puntos provisionales de corte para la disminución de la masa muscular en ancianos de Caldas a partir de los datos de la composición muscular esquelética de jóvenes entre los 18 y los 35 años mediante el análisis de bioimpedancia eléctrica, con lo cual se podría hacer el diagnóstico de sarcopenia en esta región.

\section{Materiales y métodos}

El estudio de tipo descriptivo y transversal se llevó a cabo con los voluntarios que aceptaron participar. Se hizo una convocatoria general por redes sociales y directamente entre estudiantes de diferentes universidades de Manizales en la cual se explicaban los objetivos del estudio. A quienes aceptaron participar, se les envió una lista de las condiciones para su inclusión y quienes cumplieron con ellas fueron citados al Laboratorio de Sarcopenia de la Universidad de Caldas, donde diligenciaron el formato de consentimiento informado y personal de enfermería debidamente entrenado les tomó las mediciones.

Los criterios de inclusión fueron: tener entre 18 y 35 años de edad; no estar hospitalizados; un espacio de vida con valor de 3 o más, que implica, en términos operativos, que el individuo 
tiene posibilidad de desplazarse por sí mismo hasta el sitio de valoración; tener una valoración global subjetiva clasificada como de persona "bien nutrida", y haber firmado el consentimiento informado.

Entre los criterios de exclusión se consideró la presencia de una enfermedad inflamatoria crónica asociada con aumento de las citocinas (como cáncer, enfermedades autoinmunitarias, demencia o enfermedad pulmonar obstructiva crónica) o que produjera afectación funcional, según lo registrado en la historia clínica. Además, se tuvieron en cuenta los antecedentes de enfermedad aguda en los tres meses anteriores; el uso de medicamentos que pudieran alterar las mediciones (diuréticos, hormonas, bloqueadores de los canales de calcio, dihidropiridínicos, anorexígenos, gabapentina en dosis de más de $1.800 \mathrm{mg} /$ día o betabloqueadores iniciados en el mes anterior); estar en un programa de entrenamiento físico vigoroso (más de seis horas por semana); amputaciones o alteraciones de la geometría corporal; lesiones o anormalidades en la piel que pudieran afectar los resultados de la bioimpedancia eléctrica (quemaduras, infección activa, hiperqueratosis u otras según criterio del personal entrenado); embarazo evidente o posible según la fecha de la última menstruación; presencia de marcapasos, prótesis de silicona u otro dispositivo que afectara la bioimpedancia eléctrica, y trastornos hidroelectrolíticos causados por falla cardíaca, mixedema, linfedema, o enfermedad renal crónica en hemodiálisis o diálisis peritoneal.

Una vez verificados los criterios de inclusión y exclusión, y recolectado el consentimiento informado, se procedió con el análisis de bioimpedancia eléctrica. Las mediciones se hicieron temprano en la mañana, en ayunas y con la vejiga urinaria vacía. La humedad y la temperatura ambiente relativas se controlaron con un calentador eléctrico (BFH416 ${ }^{\mathrm{TM}}$, Bionaire) y un deshumidificador (BMD100 ${ }^{\mathrm{TM}}$, Bionaire). La humedad relativa y la temperatura ambiental se midieron con un termohigrómetro $\left( \pm 0,1{ }^{\circ} \mathrm{C}\right)$ (13307'TM, DeltaTrak).

Se verificó que los voluntarios cumplieran con los requisitos antes de las mediciones. Las voluntarias jóvenes fueron evaluadas en periodos diferentes a los de su menstruación. La altura se midió en bipedestación luego de una espiración y con los pies descalzos, mediante un estadiómetro previamente calibrado $( \pm 0,01 \mathrm{~cm})$ (Heightronic-235, Seca $\left.{ }^{\mathrm{TM}}\right)$. El peso se midió luego de haber evacuado la vejiga y sin más ropa que una bata hospitalaria $( \pm 0,1 \mathrm{~kg})$ (PP2000, Icob-Detecto ${ }^{\mathrm{TM}}$ ). Ambas medidas se tomaron dos veces y se hizo una tercera medición cuando se encontraba una diferencia mayor de 0,5 $\mathrm{cm}$ en la talla o de $0,1 \mathrm{~kg}$ en el peso.

La bioimpedancia eléctrica se midió en el lado dominante del cuerpo, en tres ocasiones y sobre una superficie no conductora, con un equipo Hydra 4200 ${ }^{\mathrm{TM}}$ (Xitron Technology).

Para la estimación de la masa muscular esquelética, se utilizaron los datos a $50 \mathrm{kHz}$, empleando una ecuación predictiva y validada para la población hispana tomada del estudio de Janssen (17): masa muscular esquelética $(\mathrm{kg})=$ $\left[\left(T^{2} / R \times 0,401\right)+(\right.$ sexo $\times 3,825)+($ edad $\left.\times-0,071)\right]$ $+5,102$, donde $T$ es la talla en centímetros, $R$ la resistencia de la bioimpedancia eléctrica en ohms, el sexo femenino equivale a 0 y el masculino a $1, y$ la edad se registra en años.

A continuación, se calculó el índice de masa muscular esquelética ajustando la masa muscular a la talla. A los valores de dicho índice diferenciados por sexo, se les aplicó la prueba de Kolmogorov-Smirnov para determinar si había distribución normal de los datos, y se calcularon el promedio y las desviaciones estándar del índice de masa muscular esquelética con el programa SPSS ${ }^{\text {TM }}$, versión 24. A las medias del índice de masa muscular esquelética ajustadas por sexo, se les restaron dos desviaciones estándar para establecer el punto de corte para un índice bajo de masa muscular esquelética.

\section{Consideraciones éticas}

El presente estudio obtuvo la aprobación del Comité de Bioética de la Universidad de Caldas, mediante el Acta № 011 del 9 de septiembre de 2015.

\section{Resultados}

Se obtuvieron los datos de 255 voluntarios jóvenes sanos, $33,33 \%$ de los cuales correspondió a mujeres (85 participantes) y 66,67 \% a hombres (170 participantes). La edad promedio fue de $22,4 \pm 3,2$ y de $21,6 \pm 3,6$ años, y el índice de masa corporal promedio fue de $24,1 \pm 3,8$ y $22,2 \pm 3,4 \mathrm{~kg} /$ $\mathrm{m}^{2}$ para mujeres y hombres, respectivamente. El $91,7 \%$ de los participantes era originario de Caldas (cuadro 1).

Según la prueba de Kolmogorov-Smirnov, los datos del índice de masa muscular esquelética categorizados por sexo tuvieron una distribución 
Cuadro 1. Características de la población

\begin{tabular}{|c|c|c|c|}
\hline Características & $\begin{array}{c}\text { Total } \\
(\mathrm{N}=255)\end{array}$ & $\begin{array}{l}\text { Hombres } \\
(n=170)\end{array}$ & $\begin{array}{c}\text { Mujeres } \\
(n=85)\end{array}$ \\
\hline $\begin{array}{l}\text { Originarios de } \\
\text { Caldas }^{*}(\%)\end{array}$ & $234(91,7)$ & $165(97)$ & $69(81,1)$ \\
\hline $\begin{array}{l}\text { Edad promedio } \\
\text { (años) }\end{array}$ & 21,84 & 21,56 & 22,4 \\
\hline Peso promedio $(\mathrm{kg})$ & 63,27 & 64,63 & 60,55 \\
\hline Talla promedio (m) & 1,66 & 1,70 & 1,58 \\
\hline $\begin{array}{l}\text { IMC promedio } \\
\left(\mathrm{kg} / \mathrm{m}^{2}\right)\end{array}$ & 22,84 & 22,22 & 24,10 \\
\hline MME promedio (kg) & 26,76 & 30,08 & 20,12 \\
\hline $\begin{array}{l}\text { IMME promedio } \\
\left(\mathrm{kg} / \mathrm{m}^{2}\right)\end{array}$ & 9,58 & 10,35 & 8,02 \\
\hline Obesidad (\%) & 5,49 & 3,53 & 9,41 \\
\hline Sobrepeso (\%) & 19,22 & 14,71 & 28,24 \\
\hline Peso normal (\%) & 67,06 & 71,76 & 58,82 \\
\hline Bajo peso (\%) & 8,23 & 10 & 3,53 \\
\hline $\begin{array}{l}\text { Punto de corte para } \\
\text { sarcopenia }\left(\mathrm{kg} / \mathrm{m}^{2}\right)\end{array}$ & & 8,39 & 6,42 \\
\hline
\end{tabular}

* El porcentaje se estableció con respecto a la población total, al total de hombres y al total de mujeres.

IMC: índice de masa corporal; MME: masa muscular esquelética; IMME: índice de masa muscular esquelética

Cuadro 2. Criterios para el diagnóstico de sarcopenia según diferentes poblaciones de referencia

\begin{tabular}{|c|c|c|}
\hline \multicolumn{2}{|c|}{ Población de referencia } & \multirow{2}{*}{$\frac{\text { Sarcopenia }\left(\mathbf{k g} / \mathbf{m}^{2}\right)}{8,39}$} \\
\hline Caldas & Hombres & \\
\hline & Mujeres & 6,42 \\
\hline \multirow[t]{2}{*}{ NHANES III ${ }^{1}$} & Hombres & 8,50 \\
\hline & Mujeres & 5,75 \\
\hline \multirow[t]{2}{*}{ Taiwán² } & Hombres & 8,87 \\
\hline & Mujeres & 6,42 \\
\hline \multirow[t]{2}{*}{ Australia $^{3}$} & Hombres & 9,50 \\
\hline & Mujeres & 7,00 \\
\hline \multirow[t]{2}{*}{ España ${ }^{4}$} & Hombres & 8,25 \\
\hline & Mujeres & 6,68 \\
\hline
\end{tabular}

${ }^{1}$ Datos de Janssen, et al. (18)

2 Datos de Chien, et al. (15)

${ }^{3}$ Datos de Fiatarone-Singh, et al. (19)

${ }^{4}$ Datos de Masanes, et al. (20)

normal. El promedio del índice en mujeres jóvenes sanas fue de $8,02 \pm 0,80 \mathrm{~kg} / \mathrm{m}^{2}$ y de $10,35 \pm 0,98 \mathrm{~kg} /$ $\mathrm{m}^{2}$ en hombres.

Con base en estos resultados y la definición de sarcopenia como un índice de masa muscular esquelética por debajo de dos desviaciones estándar de la media de dicho índice en la población de referencia, el punto de corte fue de $6,42 \mathrm{~kg} / \mathrm{m}^{2}$ para las mujeres y de $8,39 \mathrm{~kg} / \mathrm{m}^{2}$ para los hombres.

\section{Discusión}

La sarcopenia es un importante problema de salud en geriatría, sin embargo, no se había establecido en nuestro medio un punto de corte para definir un bajo índice de masa muscular. En el presente estudio, se propuso definir la sarcopenia en ancianos como un índice de masa muscular esquelética inferior a $6,42 \mathrm{~kg} / \mathrm{m}^{2}$ en mujeres y, a $8,39 \mathrm{~kg} / \mathrm{m}^{2}$ en hombres.

En diversos estudios se han establecido los puntos de corte para la sarcopenia en diferentes poblaciones y con distintos métodos de medición de la masa muscular; sin embargo, los resultados son variables, lo que corrobora la idea de que no existe una población universal de referencia (16).

Al comparar los datos de nuestra población con los obtenidos de la cohorte de la National Health and Nutrition Examination Survey III (NHANES III) (18), en la cual el punto de corte para sarcopenia se estableció como un índice de masa muscular esquelética de $8,50 \mathrm{~kg} / \mathrm{m}^{2}$ en hombres y de $5,75 \mathrm{~kg} / \mathrm{m}^{2}$ en mujeres con base en el riesgo de discapacidad física, se observó que la masa muscular fue mucho menor en mujeres estadounidenses que en la población del presente estudio, lo que implicaría que el uso de los datos de la NHANES III tendería a subestimar la prevalencia de sarcopenia en nuestro medio. La composición corporal esquelética en los hombres evidenció que el índice de masa muscular esquelética en la población estadounidense fue ligeramente mayor que en la cohorte de personas jóvenes del presente estudio.

Al comparar con los datos correspondientes a una población asiática, específicamente de Taiwán, en la cual el punto de corte para sarcopenia se definió como un índice de masa muscular esquelética menor de $8,87 \mathrm{~kg} / \mathrm{m}^{2}$ en hombres y de $6,42 \mathrm{~kg} / \mathrm{m}^{2}$ en mujeres (15), pudo constatarse que fue igual al de las mujeres de esta población colombiana, pero mayor que el de los hombres. Fiatarone, et al. (19), establecieron como punto de corte para la sarcopenia en la población australiana un índice de masa muscular esquelética menor de $9,5 \mathrm{~kg} /$ $\mathrm{m}^{2}$ en hombres y de $7,00 \mathrm{~kg} / \mathrm{m}^{2}$ en mujeres, lo que, comparado con los datos del presente estudio, es ligeramente mayor tanto en hombres como en mujeres. 
En una población española, el grupo de Masanes estableció que los valores del índice de masa muscular esquelética por debajo de $8,25 \mathrm{~kg} / \mathrm{m}^{2}$ para hombres y de $6,68 \mathrm{~kg} / \mathrm{m}^{2}$ para mujeres, correspondían a sarcopenia (20), datos un poco más cercanos a los obtenidos en el presente estudio, aunque el índice fue menor en hombres y mayor en mujeres.

En un estudio en México (21) y en otro en Chile (22), se establecieron puntos de corte para la sarcopenia que no son comparables con los de este estudio, ya que la técnica usada en ambos fue la absorciometría con rayos $X$ de energía dual y no el análisis de bioimpedancia eléctrica. Estos datos demuestran cómo los puntos de corte para sarcopenia difieren según la técnica usada y la población de referencia de la cual se derivan (cuadro 2), y evidencian la importancia de contar con puntos de corte específicos para la población en la cual se utilizarán.

Si bien el consenso EWGSOP propone los puntos de corte para definir como bajo un índice de masa muscular esquelética mediante el análisis de bioimpedancia eléctrica en una cohorte americana (NHANES III) y otro estudio en una asiática (Taiwán) (9), ello podría generar dificultades para estimar la prevalencia de sarcopenia en Colombia, pues, como lo han demostrado otros autores (23), los datos varían dependiendo de la población de referencia.

Hasta donde se sabe, este es el primer estudio en el que se establece un índice de masa muscular esquelética bajo a partir de la composición muscular esquelética de jóvenes colombianos medida por bioimpedancia eléctrica, lo que implica un avance inicial en aras de la precisión diagnóstica de la sarcopenia, y permite una mejor estimación del problema en la población anciana de la región centro-andina. Además, los datos del presente trabajo avalan parcialmente lo propuesto por algunos autores en cuanto a que la población latina podría tener una composición corporal similar a la de la población asiática (en este caso, la de las mujeres) y distinta a la norteamericana (24).

Entre las limitaciones del estudio, debe mencionarse el tamaño de la muestra, especialmente la de mujeres, el cual podría ser insuficiente y no representativo de la población. Sin embargo, se considera que, dada la ausencia de datos, la información obtenida en el presente estudio puede ser un importante referente para estudios ulteriores. Dado que la mayoría de la población era originaria de Caldas, los datos podrían no ser aplicables en su totalidad a poblaciones diferentes a la de la zona andina o a poblaciones como los indígenas y las negritudes.

Otra limitación podría ser que no se contó con un método de referencia más avanzado, como la absorciometría con rayos $X$ de energía dual o las imágenes de resonancia magnética, debido a los altos costos que ello implica. Por último, es posible que los resultados no sean aplicables a poblaciones medidas con otros instrumentos de bioimpedancia, comparación que no se contempló en los objetivos iniciales del estudio, o con métodos diferentes a los de bioimpedancia.

En conclusión, este es el primer estudio que ofrece una descripción de la composición corporal muscular esquelética en jóvenes en Colombia, especialmente de la zona andina, y propone puntos de corte para la sarcopenia en la población anciana en nuestro medio. Si bien el tamaño de la muestra de mujeres jóvenes podría ser una limitante para la generalización de los datos, se trata de una primera aproximación al problema de la falta de criterios propios para establecer dicho diagnóstico. Se requieren estudios futuros en otras poblaciones colombianas para establecer los criterios del diagnóstico de sarcopenia a nivel nacional.

\section{Agradecimientos}

A los voluntarios jóvenes que participaron en el presente estudio.

\section{Conflicto de intereses}

Los autores del presente estudio declaran no tener conflictos de tipo financiero, personal o de cualquier otra índole, con respecto a este trabajo.

\section{Financiación}

El estudio fue financiado con recursos de la Universidad de Caldas.

\section{Referencias}

1. Rosenberg IH. Sarcopenia: Origins and clinical relevance. J Nutr. 1997;127:990S-1. https://doi.org/10.1016//j.cger. 2011.03.003

2. Evans WJ, Campbell WW. Sarcopenia and age-related changes in body composition and functional capacity. J Nutr. 1993;123:465-8. https://doi.org/10.1093/jn/123.suppl_2.465

3. Cederholm TE, Bauer JM, Boirie Y, Schneider SM, Sieber CC, Rolland Y. Toward a definition of sarcopenia. Clin Geriatr Med. 2011;27:341-53. https://doi.org/10.1016/j. cger.2011.04.001 
4. Fantin F, Di Francesco V, Fontana G, Zivelonghi A, Bissoli L, Zoico E, et al. Longitudinal body composition changes in old men and women: Interrelationships with worsening disability. J Gerontol A Biol Sci Med Sci. 2007;62:1375-81. https://doi.org/10.1093/gerona/62.12.1375

5. Fielding RA, Vellas B, Evans WJ, Bhasin S, Morley JE, Newman AB, et al. Sarcopenia: An undiagnosed condition in older adults. Current consensus definition: Prevalence, etiology, and consequences. International Working Group on Sarcopenia. J Am Med Dir Assoc. 2011;12:249-56. https://doi.org/10.1016/j.jamda.2011.01.003

6. Janssen I. The epidemiology of sarcopenia. Clin Geriatr Med. 2011;27:355-63. https://doi.org/10.1016/j.cger.2011. 03.004

7. St-Onge MP, Gallagher D. Body composition changes with aging: The cause or the result of alterations in metabolic rate and macronutrient oxidation. Nutrition. 2010;26:152-5. https://doi.org/10.1016/j.nut.2009.07.004

8. Gómez-Cabello A, Vicente-Rodríguez G, Vila-Maldonado S, Casajús JA, Ara I. Aging and body composition: The sarcopenic obesity in Spain. Nutr Hosp. 2012;27:22-30. https://doi.org/10.1590/S0212-16112012000100004

9. Cruz-Jentoft AJ, Baeyens JP, Bauer JM, Boirie Y, Cederholm T, Landi F, et al. Sarcopenia: European consensus on definition and diagnosis/Report of the European Working Group on Sarcopenia in Older People. Age Ageing. 2010;39:412-23. https://doi.org/10.1093/ ageing/afq034

10. Muscaritoli M, Anker SD, Argilés J, Aversa Z, Bauer JM, Biolo G, et al. Consensus definition of sarcopenia cachexia and pre-cachexia: Joint document elaborated by Special Interest Groups (SIG) "cachexia-anorexia in chronic wasting diseases" and "nutrition in geriatrics". Clin Nutr. 2010;29:154-9. https://doi.org/10.1016/j.clnu.2009.12.004

11. Walrand S, Guillet C, Salles J, Cano N, Boirie Y. Physiopathological mechanism of sarcopenia. Clin Geriatr Med. 2011;27:365-85. https://doi.org/10.1016/j.cger.2011. 03.005

12. Woodrow G. Body composition analysis techniques in the aged adult: Indications and limitations. Curr Opin Clin Nutr Metab Care. 2009;12:8-14. https://doi.org/10.1097/ MCO.0b013e32831b9c5b

13. Müller MJ, Geisler C, Pourhassan M, Glüer CC, BosyWestphal A. Assessment and definition of lean body mass deficiency in the elderly. A Eur J Clin Nutr. 2014;68:1220-7. https://doi.org/ 10.1038/ejcn.2014.169

14. Janssen I, Heymsfield SB, Ross R. Low relative skeletal muscle mass (sarcopenia) in older persons is associated with functional impairment and physical disability. J Am Geriatr Soc. 2002;50:889-96. https://doi. org/10.1046/j.1532-5415.2002.50 216.x

15. Chien MY, Huang TY, Wu YT. Prevalence of sarcopenia estimated using a bioelectrical impedance analysis prediction equation in community-dwelling elderly people in Taiwan. J Am Geriat Soc. 2008;56:1710-5. https://doi. org/10.1111/j.1532-5415.2008.01854.x

16. Pagotto V, Silveira EA. Applicability and agreement of different diagnostic criteria for sarcopenia estimation in the elderly. Arch Gerontol Geriatr. 2014;59:288-94. https://doi. org/10.1016/j.archger.2014.05.009

17. Janssen I, Heymsfield SB, Baumgartner RN, Ross R. Estimation of skeletal muscle mass by bioelectrical impedance analysis. J Appl Physiol. 2000;89:465-71. https://doi.org/10.1152/jappl.2000.89.2.465

18. Janssen I, Baumgartner RN, Ross R, Rosenberg IH, Roubenoff R. Skeletal muscle cutpoints associated with elevated physical disability risk in older men and women. Am J Epidemiol. 2004;159:413-21. https://doi.org/10.1093/ aje/kwh058

19. Fiatarone-Singh MA, Singh NA, Hansen RD, Finnegan TP, Allen BJ, Diamond TH, et al. Methodology and baseline characteristics for the sarcopenia and hip fracture study: A 5-year prospective. J Gerontol A Biol Sci Med Sci. 2009;64:568-74. https://doi.org/10.1093/gerona/glp002

20. Masanes F, Culla A, Navarro-González M, Navarro-López M, Sacanella E, Torres B, et al. Prevalence of sarcopenia in healthy community-dwelling elderly in an urban area of Barcelona (Spain). J Nutr Health Aging. 2012;16:184-7. https://doi.org/10.1007/s12603-011-0108-3

21. Alemán-Mateo H, Ruiz-Valenzuela RE. Skeletal muscle mass indices in healthy young Mexican adults aged 20-40 years: Implications for diagnoses of sarcopenia in the elderly population. Scientific World Journal. 2014;2014:672158. https://doi.org/10.1155/2014/672158

22. Lera L, Ángel B, Sánchez H, Picrin Y, Hormazabal MJ, Quiero A, et al. Validation of cut points of skeletal muscle mass index for identifying sarcopenia in Chilean older people. Nutr Hosp. 2014;31:1187-97. https://doi. org/10.3305/nh.2015.31.3.8054

23. González-González DR, López-Salazar AM, GonzálezCorrea CH. Resultados preliminares de un estudio sobre prevalencia de sarcopenia en ancianos. Rev Asoc Colomb Gerontol Geriatr. 2015;29:2023-33.

24. Alberti KG, Zimmet P, Shaw J. Metabolic syndrome-a new world-wide definition. A Consensus Statement from the International Diabetes Federation. Diabet Med. 2006;23:46980. https://doi.org/10.1111/j.1464-5491.2006.01858.x 